\title{
In-situ Transmission Electron Microscopy (TEM) Study on the Lithium Ion Transport in Si-Ge heterostructured Nanowires
}

\author{
Yang Liu ${ }^{1}$, Xiao Hua Liu ${ }^{1}$, Binh-Minh Nguyen ${ }^{2,3}$, Jinkyoung Yoo ${ }^{2}$, John P. Sullivan ${ }^{4}$, S. Tom Picraux ${ }^{2}$, \\ and Shadi A. Dayeh ${ }^{2,3}$ \\ 1. Center for Integrated Nanotechnologies, Sandia National Laboratories, Albuquerque, New Mexico \\ 87185, USA \\ 2. Center for Integrated Nanotechnologies, Los Alamos National Laboratory, Los Alamos, New Mexico \\ 87545, USA \\ 3. Department of Electrical and Computer Engineering, University of California San Diego, La Jolla, \\ California 92093, USA \\ 4. Materials Physics Department, Sandia National Laboratories, Livermore, California 94551, USA
}

Controlling the transport of lithium (Li) ions and their reaction with electrodes is critically important in the design of Li-ion batteries for achieving high capacity, high rate capability and long lifetime. One straightforward application is to mitigate the mechanical failure of battery electrode by controlled modification of the volume expansion direction. In the past two decades, it has been shown that ionic transport properties can be dominated by interfaces at the nanoscale $[1,2]$. Heterojunctions (namely chemical and structural discontinuities) in nanostructures provide the possibility to control the Li diffusion pathways, resulting in customizing volume expansion behavior. The flexibility in composition and structure enabled by tailoring electrodes at the nanoscale could drastically change the ionic transport and help meet new levels of Li-ion battery performance.

Recently, the in-situ transmission electron microscopy (TEM) electrochemistry technique has emerged as a powerful approach to understand lithiation behaviors and reaction mechanisms during battery operation, attributed to the unique capability of dynamically monitoring the structural evolution, phase transformation, and chemical composition change of electrodes with high resolution [3, 4]. Si-Ge composite anodes have the advantage of integrating a balanced and enhanced performance in Li-ion batteries due to the combination of the high capacity of Si and excellent rate capability of Ge. In this presentation, Li ion transport in $\mathrm{Si}-\mathrm{Ge}$ heterostructures (i.e. Ge/Si core/shell and $\mathrm{Si} / \mathrm{Ge}$ core/shell nanowires) was systematically studied inside the TEM in real-time. In sharp contrast to the radial lithiation behavior in pure Ge and pure $\mathrm{Si}$ nanowires as well as in $\mathrm{Si} / \mathrm{Ge}$ core/shell nanowires, pure axial lithiation was observed in $\mathrm{Ge} / \mathrm{Si}$ core/shell nanowires. These striking observations validate that radial heterostructuring ( $\mathrm{Si}$ shell on Ge core) can suppress the commonly observed surface insertion of Li ions that has been reported in all nanoscale systems to date, and exclusively induce axial lithiation along the $<111>$ direction in a layer-by-layer fashion. The deposition of a conformal, epitaxial, and ultrathin Si shell $(1 \sim 5 \mathrm{~nm})$ on Ge nanowires creates an effective chemical potential barrier for Li ion diffusion through the surface, allowing only axial lithiation and volume expansion. A set of control experiments, such as lithiation of $\mathrm{Ge} / \mathrm{SiOx}$ core/shell nanowire, carbon-coated $\mathrm{Ge} / \mathrm{Si}$ core/shell nanowire and $\mathrm{Ge} / \mathrm{Si}$ core/shell nanowire after etching of surface Si layer, were also conducted to demonstrate the interface and bandgap engineering effect on ionic transport properties. Our work [5] is the first direct observation of the dramatic interfacial effect on ionic transport at the nanoscale. Specifically, we demonstrate for the first time that interface and bandgap engineering of electrochemical reactions can be utilized to control the nanoscale ionic transport/insertion paths, which provides a powerful additional degree of freedom to define the electrochemical reactions in Li-ion batteries [6]. 


\section{References:}

[1] J. Maier, Nat. Mater. 4 (2005), 805.

[2] J. Maier, Solid State Ionics 175 (2004), 7.

[3] X. Liu and J. Huang, Energy Environ. Sci. 4 (2011), 3844.

[4] X. Liu et al, Adv. Energy Mater. 2 (2012), 722.

[5] Y. Liu et al, Nano Letters 13 (2013), 4876.

[6] This work was supported by the NEES EFRC project, LDRD funding, and was performed, in part, at the Center for Integrated Nanotechnologies, a U.S. DOE, Office of Basic Energy Sciences user facility at Los Alamos National Laboratory (Contact DE-AC52-06NA25396) and Sandia National Laboratories (Contract DE-AC04-94AL85000). S. A. Dayeh acknowledges the faculty start-up funds at UCSD.

Sandia National Laboratories is a multi-program laboratory managed and operated by Sandia Corporation, a wholly owned subsidiary of Lockheed Martin Corporation, for the U.S. Department of Energy's National Nuclear Security Administration under contract DE-AC04-94AL85000.
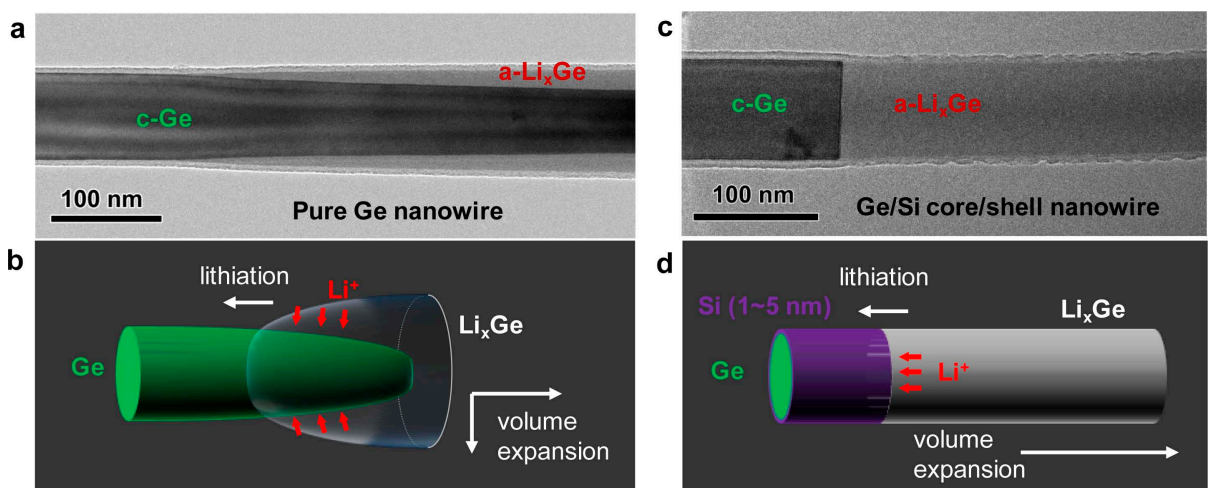

Figure 1. Sharp contrast of radial lithiation of pure Ge nanowire $(a, b)$ and axial lithiation of $\mathrm{Ge} / \mathrm{Si}$ core/shell nanowire (c, d).

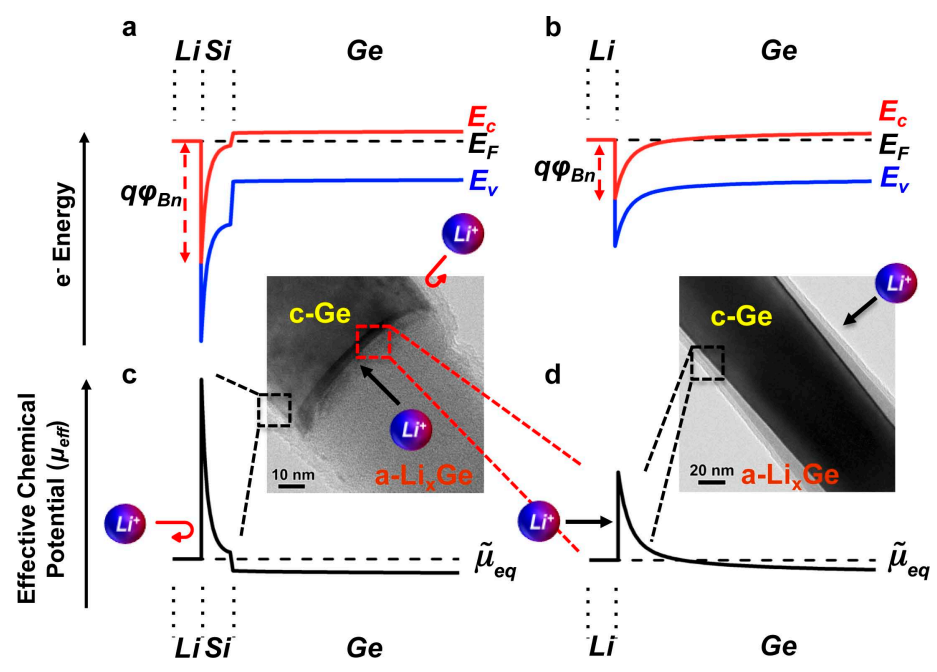

Figure 2. Comparison of band-edge profile and effective $\mathrm{Li}$ ion potential in $\mathrm{Ge} / \mathrm{Si}$ core/shell nanowire (a, c) and pure Ge nanowire (b, d). 